\title{
Distal Deep Vein Thrombosis in a Patient Cured of Severe COVID-19 Pneumonia at Jimma, Oromia, Ethiopia 2021: A Rare Case Report
}

\author{
Dejene Debela Snr (D) ${ }^{1-3}$ \\ Elias Ababulgu 4 \\ Gaddisa Desu' ${ }^{1,4}$ \\ Serkaddis Abebe' \\ Tesfaye Adugna ${ }^{1,4}$ \\ 'Department of Internal Medicine, \\ Fromsis Primary Hospital (FPH), Jimma, \\ Oromia, Ethiopia; ${ }^{2}$ Department of \\ Quality Improvement Unit, Shenen Gibe \\ General Hospital, Jimma, Oromia, \\ Ethiopia; ${ }^{3}$ Department of Center for \\ Innovative Drug Development and \\ Therapeutic Trials for Africa (CDT- \\ Africa), College of Health Sciences, Addis \\ Ababa University, Addis Ababa, Ethiopia; \\ ${ }^{4}$ Jimma University Medical Center \\ (JUMC), Jimma, Oromia, Ethiopia
}

\begin{abstract}
Background: Deep vein thrombosis (DVT) is commonly identified and diagnosed in the emergency department. Factors including sedentary life (immobility), pregnancy in women, cancer, postoperation, admission to ICU, smoking, and obesity are identified risks for thrombosis development. We report a case of a 35-year-old man who presented to the emergency department developing left lower leg swelling and pain, low-grade fever, and headache after he was treated and discharged, cured of severe COVID-19. Then venous and arterial Doppler ultrasound of the lower leg revealed dilated, absent flow and luminal thrombus in the distal popliteal, anterior and posterior tibial veins and perforator vessels were diagnosed as leg DVT.

Conclusion: DVT is a hematological emergency that needs serious consideration in prevention as well early diagnosis in patients with possible risk factors. This case report aims to arouse the clinician's awareness of the occurrence of deep vein thrombosis during and after COVID-19.
\end{abstract}

Keywords: post-COVID-19, thromboembolism, hypercoagulability, antithrombotic drugs

\section{Introduction}

Deep vein thrombosis (DVT) is the formation of one or more blood clots in one of the body's large veins, most commonly in the lower limbs. ${ }^{1}$ Several risk factors have been specifically studied and associated with venous thrombotic events. ${ }^{2}$ DVT is high in postoperative patients, ${ }^{3}$ older ages, ${ }^{4}$ hypertensive, ${ }^{5}$ admitted to ICU, and insertion of central venous catheters. ${ }^{6}$ Increases in the inflammatory response, hypoxia, immobilization, and disseminated intravascular coagulation (DIC) caused by COVID-19 are suggested mechanisms of thrombus formation. ${ }^{7}$ But no data are available regarding the time of occurrence of thromboembolism after recovering from severe COVID-19.

The complications of DVT are pulmonary embolism and adverse impact on the limb motor function of patients. ${ }^{8}$ In this report, we present a patient with DVT after recovery from COVID-19.

\section{Case Presentation}

A 35-year-old man was admitted to the emergency department of our hospital complaining of left lower leg pain and swelling, difficulty moving, headache, and low-grade fever within the last two days. He was discharged from our hospital four
Correspondence: Dejene Debela Addis Ababa, 9086, Ethiopia Tel +251913910868

Email dejene.tolossa@aau.edu.et 
days before after being treated recovering from severe COVID-19. The patient was admitted and treated with the hospital standard of care (vancomycin $1 \mathrm{~g}$ iv BID; ceftazidime $1 \mathrm{~g}$ iv BID; dexamethasone $8 \mathrm{mg}$ iv daily; oxygen; antithrombotic prophylaxis unfractionated heparin 5000 iu SC BID) for 10 days. He had no past medical history of diabetes mellitus, cardiovascular disease, hypertension, cigarette smoking, or previous deep venous thrombosis. He denied any family history of thromboembolism, bleeding, or clotting disorders. The patient did not take any other medications other than vancomycin, ceftazidime, and dexamethasone. After taking history thoroughly the patient was examined and investigated by all available laboratories and imaging. The vital signs at admission were pulse rate of 80 beats per minute, respiratory rates of 22 breaths per minute, and temperature of $36.9^{\circ} \mathrm{C}$, oxygen saturation of $93-96 \%$ with atmospheric air.

On musculoskeletal examination, the relevant physical finding was swelling of the left calf with decreased range of movement, hot to touch, and moderate tenderness and absent distal pulses. The patient was investigated with the following results. Complete blood test showed leukocytosis $(17,500 /$ $\mathrm{mm}^{3}$, normal range $<11,000 / \mathrm{mm}^{3}$ ) with lymphocytosis $\left(4.24 / \mathrm{mm}^{3}\right.$, normal range: $\left.1.3-4\right)$, and normal platelet count (241,000, normal range: $156,000-342,000)$; Coagulation profile results were prothrombin time (10.3 seconds, normal range: $10-14$ seconds), activated partial thromboplastin time (21.9 seconds, normal range: $26.1-36.3$ seconds), and international normalized ratio (0.88, normal range: $0.9-1.6)$. Renal function was in normal range (creatinine $1.21 \mathrm{mg} / \mathrm{dL}$, normal range: $<1.3 \mathrm{mg} / \mathrm{dL}$ ), fasting blood sugar (FBS $105.7 \mathrm{mg} / \mathrm{dL}$, normal range: $75-115 \mathrm{mg} / \mathrm{dL}$ ), liver function was in the normal range (ASAT/GOT 48, normal range: 0-47 U/L, ALAT/ GPT $37 \mathrm{U} / \mathrm{L}$, normal range: $<50 \mathrm{U} / \mathrm{L}$ ). Due to the clinical suspicion of acute arterial thrombosis, an urgent venous and arterial Doppler ultrasound of the lower leg was done and revealed dilated, absent flow seen and filled with luminal thrombus in the distal popliteal, anterior, and posterior tibial veins and perforator vessels. Emergency chest X-ray was also done to rule out pulmonary infection, but revealed normal lung parenchyma (Figure 1). An echocardiogram was performed and reported a normal left ventricle with preserved ejection fraction (62\%), right cavities with normal size and function, no relevant valve disease, and no pulmonary hypertension signs seen. Immediately, at the emergency department, the patient was initiated with unfractionated heparin (UFH) loading dose of $5000 \mathrm{IU}$ iv, then 12,500 IU subcutaneous BID, and then

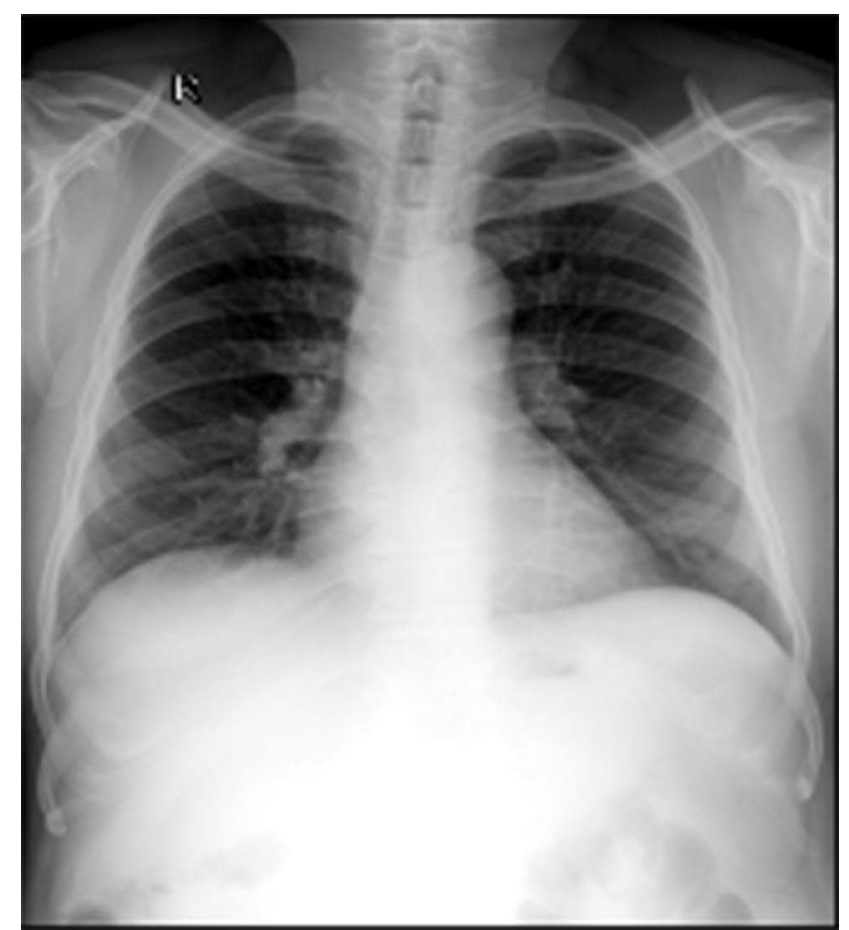

Figure I Chest X-ray on admission showed normal lung parenchyma.

transferred to the inpatient medical ward. After five days of admission, the patient was improved clinically and blood flow of the left lower leg was restored. The patient could be discharged then with warfarin $5 \mathrm{mg} \mathrm{PO}$ daily without any complications.

\section{Discussion}

Our case report described DVT in a patient who had recovered from severe COVID-19 one week earlier without thromboembolism risk factors. To the best of our knowledge, no similar presentation has been reported yet. Doppler ultrasound of the lower limb showed DVT after the patient presented with typical clinical feature of DVT. Our case report result showed that DVT can develop in patients that have recovered from severe COVID-19 and given possible prophylaxis. The patient was constantly mobile before this illness and had no trauma to his leg. Many reports showed that patients with COVID-19 complication of thromboembolism and organ failure leads to higher death rate $^{9}$ but there are few reports of DVT in patients with COVID-19. In a study by Davoudi et al, the patient presented with typical symptoms of DVT, such as swelling, redness, and tenderness and was diagnosed to have COVID-19 confirmed by RT-PCR testing. ${ }^{10} 1111$ In our case, DVT was suspected due to leg swelling and pain, fever, decreased limb mobility, and tenderness, which was 
then confirmed by color Doppler ultrasound in the distal popliteal, anterior and posterior tibial veins and perforator vessels. One study done on 128 critically ill ICU patients with COVID-19 showed DVT was also diagnosed in three patients which occurred after the diagnosis of COVID-19 due to hospitalization ${ }^{11}$ supports the results of our patient. Even though the exact cause of DVT after COVID-19 is still being studied, the possible mechanism may be that coronavirus attacks the human body through 2 -angiotensinconverting enzyme. ${ }^{12}$ The problems of blood clotting will be increased due to cytokine storms by the virus leading to DVT development. ${ }^{13}$

\section{Conclusion}

Thromboembolic events are common during and after the recovery from COVID-19 which is associated with a poor outcome. So, diagnosing DVT requires a high level of clinical suspicion and careful management with therapeutic doses of LMWH should be considered in severe COVID-19 patients.

\section{Abbreviations}

COVID-19, Coronavirus disease 2019; DVT, deep vein thrombosis; EOPD, emergency outpatient department; FBS, fasting blood sugar; TE, thromboembolism; UFH, unfractionated heparin.

\section{Ethical Approval}

We conducted the case report in compliance with the Declaration of Helsinki, good clinical practices, institutional regulatory requirements. Fromsis Primary Hospital Ethical Committee approved it.

\section{Consent for Publication}

The patient signed informed consent forms for the use of case details and images for publication and scientific purposes.

\section{Acknowledgments}

The authors would like to thank all staff of Fromsis Primary Hospital.

\section{Disclosure}

The authors report no conflicts of interest in this work.

\section{References}

1. Piazza G, Goldhaber SZ. Acute pulmonary embolism: part I: epidemiology and diagnosis. Circulation. 2006;114(2):e28-32. doi:10.1161/CIRCULATIONAHA.106.620872

2. McLendon K, Goyal A, Bansal P, Attia M Deep venous thrombosis risk factors. Treasure Island (FL): Stat Pearls Publishing; 2021. Available from: https://www.ncbi.nlm.nih.gov/books/NBK470215/. Accessed June 02, 2021.

3. Tian Q, Li M. Risk factors of deep vein thrombosis of lower extremity in patients undergone gynecological laparoscopic surgery: what should we care. BMC Womens Health. 2021;21(1):130. doi:10.1186/ s12905-021-01276-7

4. Shi J, Ye J, Zhuang X, Cheng X, Fu R, Zhao A. Application value of Caprine risk assessment model and elevated tumor-specific D-dimer level in predicting postoperative venous thromboembolism for patients undergoing surgery of gynecologic malignancies. $J$ Obstet Gynaecol Res. 2019;45(3):657-664.

5. Laws A, Anderson K, Hu J, et al. Implementation of a venous thromboembolism prophylaxis protocol using the caprine risk assessment model in patients undergoing mastectomy. Ann Surg Oncol. 2018;25(12):3548-3555. doi:10.1245/s10434-018-6696-y

6. Davidson BL. Risk assessment and prophylaxis of venous thromboembolism in acutely and/or critically ill patients. Haemostasias. 2000;30(Suppl 2):77-81; discussion 63.

7. Klok F, Kruip M, Van der Meer N, et al. Incidence of thrombotic complications in critically ill ICU patients with COVID-19. Thromb Res. 2020;191:145-147. doi:10.1016/j.thromres.2020.04.013

8. Gutzeit O, Lauterbach R, Loberman Z, Sachner R, Karram T, Lowenstein L. Laparoscopic sacrocolpopexy complication: ilio-femoral deep vein thrombosis. Eur J Obstet Gynecol Reprod Biol. 2020;247:270-271. doi:10.1016/j.ejogrb.2020.02.040

9. Moragón-Ledesma S, Galeano-Valle F, Calleja-Cartón E, Del-ToroCervera J, Demelo-Rodríguez P. Bilateral deep vein thrombosis, vena cava agenesis, and renal abnormalities: KILT syndrome - a case report and literature review. J Cardiovasc Transl Res. 2020;13 (4):629-631. doi:10.1007/s12265-019-09935-9

10. Bikdeli B, Madhavan MV, Jimenez D, et al. COVID-19 and thrombotic or thromboembolic disease: implications for prevention, antithrombotic therapy, and follow-up: JACC state-of-the-art review. $J \mathrm{Am}$ Coll Cardiol. 2020;75(23):2950-2973. doi:10.1016/j. jacc.2020.04.031

11. Davoodi L, Jafarpour H, Taghavi M, Razavi A. COVID-19 presented with deep vein thrombosis: an unusual presenting. J Investig Med High Impact Case Rep. 2020;8:2324709620931239.

12. Zhang H, Penninger JM, Li Y, Zhong N, Slutsky AS. Angiotensinconverting enzyme 2 (ACE2) as a SARS-CoV-2 receptor: molecular mechanisms and potential therapeutic target. Intensive Care Med. 2020;46(4):586-590. doi:10.1007/s00134-020-05985-9

13. Tarannum N, Azam MS, Premchand RK. May-Thurner syndrome and recurrent DVT: a case report. Indian J Clin Cardiol. 2020;1 (1):13-16. doi:10.1177/2632463619898010 


\section{Publish your work in this journal}

The International Medical Case Reports Journal is an international, peer-reviewed open-access journal publishing original case reports from all medical specialties. Previously unpublished medical posters are also accepted relating to any area of clinical or preclinical science. Submissions should not normally exceed 2,000 words or 4 published pages including figures, diagrams and references. The manuscript management system is completely online and includes a very quick and fair peer-review system, which is all easy to use. Visit http://www.dovepress.com/testimonials.php to read real quotes from published authors. 